\title{
Detecting Particle Dark Matter Signatures via Cross-Correlation of Gamma-Ray Anisotropies and Cosmic Shear
}

\author{
Stefano Camera \\ CENTRA, Instituto Superior Técnico, Universidade de Lisboa, \\ Avenida Rovisco Pais 1, 1049-001 Lisboa, Portugal \\ email: stefano.camera@tecnico.ulisboa.pt
}

\begin{abstract}
Similarly to gravitational lensing effects like cosmic shear, cosmological $\gamma$-ray emission too is to some extent a tracer of the distribution of dark matter (DM) in the Universe. Intervening DM structures source gravitational lensing distortions of distant galaxy images, and those same galaxies can emit $\gamma$ rays, either because they host astrophysical sources, or directly by particle DM annihilations or decays occurring in the galactic halo. If such $\gamma$ rays exhibit correlation with the cosmic shear signal, this will provide novel information on the composition of the extragalactic $\gamma$-ray background.
\end{abstract}

Keywords. Dark matter, gravitational lensing, gamma rays: observations, methods: statistical

\section{Introduction}

Our current understanding of the Universe is encoded in the concordance cosmological model, which requires a large abundance of cold dark matter (DM) in the present cosmos (Planck Collaboration, 2013). However, very little is known about its nature. One of the most promising interpretations of DM sees it as a Weakly Interacting Massive Particle (WIMP). Many investigation strategies are currently being pursued to the aim of improving our knowledge on the subject. One of the theoretically more promising approaches is the search for WIMP DM signatures in the extragalactic $\gamma$-ray background (see e.g. Fornengo, Pieri \& Scopel, 2004). The underlying hypothesis is that the DM structures in the Universe can emit light at various wavelengths, including the $\gamma$-ray range. Indeed, besides $\gamma$ rays produced by astrophysical sources hosted inside DM haloes, WIMP DM itself may be a source of $\gamma$ rays through its self-annihilation (or decay, depending on the properties of the DM particle).

\section{Auto-Correlation of Gamma-Ray Anisotropies}

The most recent measurement of the extragalactic $\gamma$-ray background (EGB) was performed by Abdo et al. (2010) for the Fermi Large Area Telescope (LAT), covering a range between $200 \mathrm{MeV}$ and $100 \mathrm{GeV}$. The emission is obtained by subtracting from the whole Fermi-LAT data the contribution of resolved sources (both point-like and extended) and the Galactic foreground (due to cosmic-ray interaction with the interstellar medium). Unresolved astrophysical sources like blazars and radio galaxies still contribute to the EGB, but the exact amount of their contribution is unknown. Similarly, $\gamma$ rays produced by DM annihilation or decay in principle also contribute to the EGB.

However, the fact that the measured EGB energy spectrum is well compatible with a power law suggests that DM cannot play a leading rôle in the whole energy range (Abdo 
et al., 2010). In the angular anisotropies of the EGB emission, DM is even more subdominant. Indeed, a detection of a significant auto-correlation angular power spectrum has been recently reported for multipoles $\ell>100$, but the absence of distinctive spectral features in the signal point towards an interpretation in terms of point-like sources like blazars (Ackermann et al., 2012; Harding \& Abazajian, 2012).

\section{Cross-Correlation of Gamma-Ray Anisotropies and Cosmic Shear}

Since the study of $\gamma$-ray anisotropies does not seem, in itself, capable of detecting WIMP DM signatures, a possible solution is to cross-correlate the $\gamma$-ray signal with other tracers of the underlying DM structure. Camera et al. (2013) proposed for the first time to employ weak gravitational lensing to this purpose. Weak lensing refers to the small distortions of images of distant galaxies, produced by the distribution of matter located between galaxies and the observer (Bartelmann \& Schneider, 2000; Bartelmann, 2011). A distorted image can be parameterised in terms of convergence $\kappa$ (controlling modifications in the size of the image) and shear $\gamma$ (accounting for shape distortions). Whilst the former is a direct estimator of matter density fluctuations integrated along the line of sight, the latter is easier to measure, through correlations in the pattern of observed source ellipticities. In the flat-sky approximation, the two generate identical angular power spectra and we thus focus on the shear as an estimator of the convergence. Present and planned surveys like the Dark Energy Survey (DES) and Euclid will reconstruct two-dimensional shear maps at various redshifts, from which we can extract the auto-correlation cosmic shear power spectrum (Abbott et al., 2005; Laureijs et al., 2011; Amendola et al., 2013).

Camera et al. (2013) demonstrated that WIMP DM distinctive signatures can be detected in the cross-correlation between the cosmic shear and the EGB. The strength of this method - which can also provide novel information on the composition of the EGBresides in the fact that the shear signal is stronger for larger halo masses and most of the $\gamma$-ray emission from annihilating/decaying DM is produced in large-mass haloes as well. Thus, their cross-correlation is more significant than for the case of astrophysical sources, associated with galaxy-mass haloes. (For more details see Camera et al., 2013, and references therein.)

Since then, Shirasaki et al. (2014) measured the 2-point cross-correlation function of Fermi-LAT data and cosmic shear, as detected by the Canada-France-Hawaii Telescope Lensing Survey (Heymans et al., 2012). Their measurement is consistent with no signal and the null detection was thus used to derive constraints on the WIMP annihilation cross section. The upper limits excluded values smaller than the thermal cross section, $3 \times 10^{-26} \mathrm{~cm}^{3} / \mathrm{s}$. This is an additional proof of the potential of such a technique for indirect detection of DM.

\subsection{Tomographic Cross-Correlation of Gamma-Ray Anisotropies and Cosmic Shear}

An even more effective technique is proposed by Camera et al. (2014). It involves a tomographic approach to the study of the cross-correlation between cosmic shear and $\gamma$-ray emission. To better model the bulk of unresolved astrophysical sources, they also include the contribution of unresolved misaligned Active Galactic Nuclei (MAGNs). This component, together with that of unresolved blazars and star-forming galaxies (SFGs), is associated to a quite large cross-correlation power spectrum. However, the abundance of those classes of sources as a function of redshift or observed $\gamma$-ray energy is quite different from that of WIMP DM. Tomography takes advantage of this behaviour by computing the cross-correlation power spectrum in different redshift and energy bins. The study 
Table 1. Forecast $1 \sigma$ marginal errors on decaying DM model parameters for the fiducial values $\left\{m_{\chi},\left\langle\sigma_{a} v\right\rangle, \mathcal{A}_{B}, \mathcal{A}_{\mathrm{SFG}}, \mathcal{A}_{\mathrm{MAGN}}\right\}=\left\{100 \mathrm{GeV}, 3 \times 10^{-26} \mathrm{~cm}^{3} / \mathrm{s}, 1,1,1\right\}$.

\begin{tabular}{|cccccc|}
\hline Binning & $m_{\chi}[\mathrm{GeV}]$ & $\left\langle\sigma_{a} v\right\rangle\left[10^{-26} \mathrm{~cm}^{3} / \mathrm{s}\right]$ & $\mathcal{A}_{B}[-]$ & $\mathcal{A}_{\mathrm{SFG}}[-]$ & $\mathcal{A}_{\mathrm{M} \mathrm{AGN}}[-]$ \\
\hline- & $2.1 \times 10^{5}$ & $8.0 \times 10^{3}$ & $4.7 \times 10^{7}$ & $7.8 \times 10^{4}$ & $8.2 \times 10^{3}$ \\
$E_{\gamma}-z$ & 11 & 0.44 & $1.3 \times 10^{4}$ & $1.4 \times 10^{2}$ & 3.2 \\
\hline
\end{tabular}

of how the power spectrum changes as a function of $z$ or $E_{\gamma}$ significantly boosts the capability of the cross-correlation power spectrum to determine the composition of the EGB and thus to distinguish DM from astrophysical components.

This is particularly useful because, on the one hand, the redshift dependence of $\gamma$ rays generated by astrophysical objects is peculiarly different from what expected for DMsourced $\gamma$ rays, and, on the other hand, the energy spectrum of DM-produced $\gamma$ rays peaks at an energy proportional to the WIMP mass, whereas unresolved astrophysical sources contribute to the EGB with well defined power laws. Preliminary results have been obtained by performing a Fisher matrix analysis for the cross-correlation of $\gamma$-ray anisotropies and cosmic shear to forecast the capabilities of a weak lensing survey like DES and a $\gamma$-ray experiment as Fermi-LAT in constraining the WIMP DM mass, $m_{\chi}$, and its annihilation cross-section, $\left\langle\sigma_{a} v\right\rangle$, or its decay rate, $\Gamma_{d}$ (Camera et al., 2014). To account for the underlying ignorance on the relative abundances, the amplitudes of the one-halo power spectra of astrophysical sources are included as nuisance parameters. The cosmic shear signal is divided into 3 redshift bins of width $\Delta z=0.4$ from 0.3 to 1.5 , whilst $\gamma$-ray measurements into 6 energy bins between 1 and $300 \mathrm{GeV}$. As an example, the forecast marginal errors on annihilating DM model parameters are summarised in Table 1, without and with the energy-redshift binning to better illustrate impact of tomography. We assume the halo substructure scenario of Gao et al. (2012).

\section{Acknowledgments}

SC is funded by FCT-Portugal under Post-Doctoral No. Grant SFRH/BPD/80274/2011. SC warmly thanks Mattia Fornasa, Nicolao Fornengo and Marco Regis for allowing him to show results from a common project.

\section{References}

Abbott, T., et al. 2005, arXiv:astro-ph/0510346

Abdo, A. A., et al. 2010, Phys. Rev. Lett. 104,101101

Ackermann, M., et al. 2012, Phys. Rev. D85, 083007

Amendola, L., et al. 2013, Living Rel. Rev. 16, 6

Bartelmann, M., 2010, Class. Quant. Grav. 27, 233001

Bartelmann, M. \& Schneider, P. 2001, Phys. Rept.340, 291

Camera, S., Fornasa, M., Fornengo, N. \& Regis, M. 2013, Astrphys. J. 771, L5

Camera, S., Fornasa, M., Fornengo, N. \& Regis, M. 2014, arXiv:astro-ph:1411.4651

Fornengo, N., Pieri, L., \& Scopel, S. 2004, Phys. Rev. D70, 103529

Gao, L., et al. 2012, Mon. Not. R. Astron. Soc. 419, 1721

Harding, J. P. \& Abazajian, K. N. 2012, J. Cosmol. Astrop. Phys. 1211, 026

Heymans, C., et al. 2012, Mon. Not. R. Astron. Soc. 427, 46

Laureijs, R., et al. 2011, ESA-SRE 12

Planck Collaboration 2013, arXiv:1303.5076

Shirasaki, M., Horiuchi, S., \& Yoshida, N. 2014, arXiv:1404.5503 